\title{
Health state utility valuation in radioactive iodine- refractory differentiated thyroid cancer
}

\author{
This article was published in the following Dove Press journal: \\ Patient Preference and Adherence \\ 3 November 2015 \\ Number of times this article has been viewed
}

\author{
Beth A Fordham' \\ Cicely Kerr' \\ Hayley M de Freitas' \\ Andrew J Lloyd' \\ Karissa Johnston ${ }^{2}$ \\ Corey L Pelletier ${ }^{3}$ \\ Gabriel Tremblay ${ }^{3}$ \\ Anna Forsythe 3 \\ Bryan Mclver ${ }^{4}$ \\ Ezra EW Cohen ${ }^{5}$ \\ 'ICON Patient Reported Outcomes, \\ Oxford, UK; ${ }^{2}$ ICON Epidemiology, \\ Toronto, ON, Canada; ${ }^{3}$ Eisai Inc., NJ, \\ ${ }^{4}$ Moffitt Cancer Center, FL, ${ }^{5}$ University \\ of California San Diego Moores \\ Cancer Center, La Jolla, CA, USA
}

Correspondence: Hayley M de Freitas ICON Patient Reported Outcomes, 100 Park Drive, Milton Park, Abingdon, Oxfordshire, OXI4 4RY, UK

Tel +44 I865 320 I44

Fax +44 2031946619

Email hayley.defreitas@iconplc.com
Purpose: The aim of this study was to elicit utilities for radioactive iodine-refractory differentiated thyroid cancer (RR-DTC) and evaluate the impact of treatment response and toxicities on quality of life.

Patients and methods: RR-DTC health states were developed based on data from a previous qualitative study and iterative review by clinical experts. Following piloting, health states underwent valuation by 100 members of the UK public during time trade-off interviews. Mean utilities and descriptive distribution statistics were calculated, and a logistic regression analysis was conducted.

Results: The demographic characteristics of the study sample were generally reflective of the UK population. Clear differentiation in valuation between health states was observed. No response/stable disease had an adjusted utility value of 0.87 , with a corresponding gain of +0.04 following a treatment response and a decline of -0.35 for disease progression. Adverse events were associated with utility decrements between -0.47 (grade III diarrhea) and -0.05 (grade I/II alopecia).

Conclusion: The trade-off interviews derived utility weights show clear differentiation between RR-DTC health states in response to treatment. The values reported in this study are suitable for cost-effectiveness evaluations for new treatments in RR-DTC.

Keywords: differentiated thyroid cancer, radioactive iodine-refractory, health-related quality of life, health utility, vignette

\section{Introduction}

Differentiated thyroid cancer (DTC) is the most common form of thyroid cancer and accounts for $>80 \%$ of cases. ${ }^{1}$ More prevalent in women than men, DTC typically presents at a young age in people aged 45-54 years., ${ }^{2,3}$ DTC is generally a treatable disease with a combination of surgery, radioactive iodine therapy, and ongoing thyroid suppression/hormone replacement therapy. ${ }^{4}$ When compared with other malignancies, the prognosis for DTC is favorable with long-term (10 years) survival rates of $>90 \%{ }^{5,6}$ As a result, thyroid cancer is one of the most prevalent malignancies, with 566,708 people living with the disease in US. ${ }^{3}$ It is expected to be the fourth leading cancer diagnosis by $2030 .^{7}$ Therefore, understanding its impact on health-related quality of life (HRQL) is of particular importance. In addition, thyroid cancer survivors report significant health effects and impact on HRQL, ${ }^{8-10}$ with even "cured" DTC patients experiencing significantly impaired HRQL when compared with controls." ${ }^{9,11,12}$ The development of radioactive iodine-refractory DTC (RR-DTC) substantially changes a patient's prognosis and poses a significant therapeutic challenge. ${ }^{13}$ Treatment options are limited and 10-year survival decreases drastically to $\sim 10 \% .{ }^{6}$ Until recently, doxorubicin, which yields low response rates and is associated with a range of toxicities, was the only approved systemic therapy. ${ }^{14}$ Treatment options have expanded with the 
introduction of tyrosine kinase inhibitors and the approval of sorafenib and lenvatinib. ${ }^{15-17}$ Due to the long life expectancy of patients, targeted agents in DTC are likely to be administered over decades, and patients will be managing their treatment and any related side effects for a longer period of time. Therefore, it is very important to understand accurately the impact of toxicities on HRQL.

The longevity of this treatment highlights the need to assess a risk-to-benefit ratio, and HRQL is a key factor in treatment decisions. ${ }^{18}$ The importance of HRQL in RR-DTC is further supported by the results of a recent qualitative study, which concluded that more advanced DTC treatment phases, including RR-DTC, have a greater impact on HRQL than earlier phases. ${ }^{19}$ The long duration of DTC therapy has important implications for the economic evaluation of new treatments. These evaluations require a reliable measure of HRQL that reflects value (or utility), and utility values for various DTC health states have been published. ${ }^{20-23}$ Reimbursement agencies, including the National Institute for Health \& Care Excellence (NICE), have raised their expectations regarding the suitability of methods for collecting HRQL data. NICE states a preference for the use of the EQ-5D-3L, a generic measure of HRQL completed by patients. ${ }^{24}$ However, when such data are unavailable, other methods such as statistical mapping are recommended. Furthermore, the EQ-5D-3L may not always be the most appropriate method for measuring and valuing health effects. ${ }^{24}$ An alternative method for capturing utilities is the vignette method, where vignette descriptions of health states are valued in a time trade-off (TTO) exercise. This method is not advocated by NICE but has been utilized frequently in oncology due to the difficulties in capturing HRQL data. Despite no methodological guidelines, NICE state recommendations regarding how such data should be collected. The use of qualitative research with patients ensures that the data are rooted in patients' experience while enabling valuation by the general public, a more feasible method when considering rare diseases with a small patient population, during which the priorities of a wider society can be reflected. Thus facilitating the elicitation of HRQL, that is, utility values, for use in health economic decision models informing Health Technology Assessment (HTA). In addition, ensuring that the content of the states reflects the five dimensions of the EQ-5D-3L and that valuation methods (face-to-face TTO interviews with the general public) mirror EQ-5D-3L valuation helps to avoid limitations of the vignette methodology.

The generally favorable prognosis of DTC has led to a commonly expressed view by clinicians of it as a "good cancer" with survivors being advised to expect a normal life. ${ }^{25,26}$ However, this is not a view shared by all, and qualitative research has found that patients view this description as dismissive and not reassuring. ${ }^{26}$ This may also result in a failure to validate a patient's legitimate health concerns in the face of a malignant disease, a situation further confounded by the lack of published data reporting the precise impact of RR-DTC on HRQL, including possible impacts of any relevant toxicities and an absence of published utilities for this phase of the disease. Furthermore, results from the recent qualitative study suggested that published utility values for earlier DTC disease stages are not generalizable to RR-DTC health states due to the greater HRQL impact of more advanced treatment phases. ${ }^{19}$ This vignette study was conducted to elicit utilities for RR-DTC health states and evaluate the utility impact of specific toxicities associated with RR-DTC treatment.

\section{Patients and methods Health state development}

Health state descriptions (vignettes) were developed based on results of the previous qualitative study in DTC, which included 14 patients with RR-DTC, ${ }^{19}$ input and iterative review from clinical experts. One-to-one telephone interviews were conducted with six health care professionals (physicians and nurses) from the UK and US with experience in treating patients with RR-DTC. Interviews were conducted by an experienced researcher using a semistructured discussion guide, informed by the results of the recent qualitative study, other published DTC HRQL literature, and treatment safety data. The clinical experts were asked to focus on their experience of "typical" patients and discuss symptoms, treatmentrelated toxicities, and any impact on HRQL that they had observed in $>50 \%$ of their patients. Discussion also covered rarer cases to help contextualize their descriptions of the more typical RR-DTC patient experience. HRQL was discussed in terms of the main domains of health status described in two commonly used preference measures, the EQ-5D-3L and SF-6D. ${ }^{27,28}$ The Response Evaluation Criteria in Solid Tumors guidelines and The Common Toxicity Criteria were used to inform discussion of clinical and patient experience of types and levels of treatment response and adverse events (AEs). ${ }^{29,30}$ A list of health states, some including AEs selected by the experts as the most common and relevant to the HRQL of patients with RR-DTC, particularly those receiving tyrosine kinase inhibitors, was finalized using feedback provided by the clinical experts. The states were as follows: stable/ no response, response (partial and complete), progressive 
disease, stable/no response with grade III diarrhea, stable/ no response with grade III fatigue, stable/no response with grade III hand-foot syndrome (HFS), and stable/no response with grades I and II alopecia. In order to standardize data collection and allow comparison of HRQL decrement, the AE health states were each combined with the stable disease state. Additional AEs that were discussed included hypertension, which, despite being reported as a relatively common event, was not included due to being asymptomatic in the majority of patients, and therefore, exerts little effect on HRQL.

Draft descriptions for each of the health states were developed and aimed to describe the impact on HRQL across the main domains of health status described in the EQ-5D-3L and SF-6D. ${ }^{27,28}$ Expert clinical review critiqued the health states for clinical accuracy and relevance. Initial review and feedback were provided by further telephone interviews with the two US physicians. The health state descriptions were revised and sent to the remaining four clinical experts for review. In cases of disagreement, the level of RR-DTC experience was considered, and clinical practice was valued more highly than the involvement in clinical trials, thus to ensure the health state descriptions reflected the experience of a typical patient and not those of a patient enrolled in a clinical trial.

To ensure that the descriptors were clear and would be understood during valuation, piloting via cognitive debriefing interviews was carried out with five members of the UK general public. Pilot participants were first asked to read and rank the health states. The interviewer then used a semistructured interview guide to explore reasons for their rankings, the participants' interpretation and evaluation of the health state descriptions, and their understanding of the terms used and the differences between the health states. Following cognitive debriefing, the health state descriptions were further refined to highlight the differences between states by underlining key text in each health state that differentiated one state from another. To prevent potential misinterpretation, in the health state that included description of HFS, the phrase "skin reaction" was revised to "skin condition" to avoid confusion with an allergic reaction. Also, the example of difficulty "walking long distances" was revised to difficulty "standing or climbing stairs" to reduce ambiguity around an impact on walking ability or mobility and to prevent overlap with text used elsewhere in the stable/no response health state description to describe a more general impact on mobility.

\section{Health state valuation}

Final health states (Supplementary material) underwent valuation by participants from the general public during a face-to-face interview using a $0-100$ visual analogue scale (VAS) and a TTO valuation method. During the VAS rating exercise, the participants were asked to rate the individual health state descriptions. The titles of the health states were not stated to avoid influencing the respondents' judgement. The descriptions were presented in a random order and participants placed them on a 100 point VAS. Participants were free to place the state of "being dead" at any position on the VAS scale; however, the full health state was always placed at 100. This task familiarized the participants with the health states and the process of rating health descriptions.

The TTO method enables health utility values to be derived based upon responses to decision scenarios. ${ }^{31}$ Interviewers used a TTO board to facilitate the participants' evaluation of how many years of full health they would be prepared to trade (lose) to avoid living in the presented health state. Participants were presented with a series of choices, between (A) living in the health state for 10 years and (B) living in a state of full health for $10-$ " $x$ " years, and asked which they preferred, with $x$ varied until the participant indicated they were indifferent between the two choices. If during the VAS rating, a participant had placed a health state below the state of "dead" and confirmed they considered the health state to be worse than being dead, in the TTO valuation, the participants were presented with an alternative series of choices, between (A) living in the health state for a $x$ years followed by $10-x$ years in full health or (B) being dead. Again, $x$ was varied until the participant indicated they were indifferent between the two choices. The amount of time in full health traded to avoid living in the health state indicates its value or utility, ranging between 0 representing dead and 1 representing full health. The valuation method for states worse than dead yields negative values that can range between -9 and 0 . These were divided by 9 to rescale to between -1 and 0 .

The participants also completed a sociodemographic questionnaire, which was used for the purposes of sample description and the EQ-5D-3L.

Informed written consent was obtained from each pilot and valuation study participant. The study protocol conformed to ethical guidelines of the 1975 Declaration of Helsinki.

\section{Participants}

A total of 100 members of the UK public underwent TTO interviews to value the defined health states. These participants were members of the general UK population to represent UK societal perspectives in line with the published 
guidance for elicitation of utility values for use in health economic decision models informing HTA. ${ }^{24,32}$ They were recruited by five experienced field interviewers in different geographical UK locations (Bolton, Dunblane, Leamington Spa, Newcastle, and Sheffield).

Eligibility criteria included $18+$ years of age, currently resident in the UK, able to understand the survey as judged by the investigator, and provided informed consent. Participants with an acute illness or cognitive impairment that may interfere with the study requirements were excluded.

\section{Analyses}

Mean TTO utilities and descriptive distribution statistics were calculated for each health state from the interview data. A regression analysis was conducted during which utilities were first transformed using a logistic function $\left(-\log \frac{1-\text { utility }}{\text { utility }}\right)$, which resulted in an empiric distribution resembling a normal distribution. In order to calculate the transformation, negative values were set to 0.02 , and values of 1.0 were set to 0.98 . The incremental impact of health states was estimated using a multivariable mixed effects model, which estimated the impact of health states relative to a base state of stable disease with no AEs. The following additional explanatory sample variables were included in the full mixed effects model: age, sex, employment status, educational qualification status, marital status, and self-reported EQ-5D-3L domain scores (moderate or extreme problem vs no problem). Adjusted predicted utilities were calculated with the sample variables found to significantly predict utility set to UK normative values. These significant sample variables reported EQ-5D$3 \mathrm{~L}$ usual activity and anxiety/depression domain scores and educational qualification status.

\section{Results}

\section{Participants}

The demographic characteristics were generally reflective of the UK population (Table 1). ${ }^{33}$ Minor discrepancies in the study sample included higher proportions of students, those who had completed university, and married individuals. The sample was predominantly of white ethnicity $(92 \%)$, which is representative of the general UK population; however, there was an absence of participants of black ethnicity. EQ-5D-3L data suggest that the study sample was healthier and reported fewer health-related problems than the general population. ${ }^{34}$ This is likely to reflect that individuals with ongoing health problems may be less likely to participate in this type of research study, a finding consistent with previous vignette studies. ${ }^{35}$

\section{Observed utilities for RR-DTC health} states

Mean utility values derived from the TTO interviews indicate how participants in the study differentiated between the RR-DTC health states (Table 2). As demonstrated by no overlap in the $95 \%$ confidence intervals (CIs), for the health states that included a grade III AE (diarrhea, fatigue, or HFS), mean utility values were significantly lower than for the no response/stable health state. However, the no response/stable and the fatigue $\mathrm{AE}$ states were separated by only 0.0001 (lower $95 \%$ CI no response/stable state: 0.7663 ; upper $95 \% \mathrm{CI}$ fatigue state: 0.7662 ). The mean utility value for no response/stable with grades I and II alopecia was also lower than no response/stable, although this difference was not statistically significant.

When comparing the results from the VAS exercise and TTO interviews, the health state ranking from "best" to "worst" shows logical ordering and consistency. All health states containing descriptions of an $\mathrm{AE}$ were rated as worse than the no response/stable health state, with grade III diarrhea rated as the lowest state.

During the TTO interviews, spontaneous comments made by participants that were relevant to the valuation of the health states were documented. These comments suggest that the health states were interpreted meaningfully and provide further context to the study results. When considering the stable/no response state, one participant felt this state was "not too bad" and another summarized the state as "Only have pain sometimes, not too many awful symptoms". A key feature of the response health state was regarding the improvement in the condition, "you are getting better". The severity of progressive disease was recognized, in particular, the depressed mood, pain, and distress related to the deterioration in health. Participants commented that they "Don't like pain, low mood and distress" and "it's getting worse so give up, no point continuing".

With regard to the AEs, alopecia was generally thought to not have a large impact on the quality of life due to no effect on functioning. Comments like "Can still work" and "Could live without my hair" exemplify this opinion. On contemplating grade III fatigue, some participants thought that fatigue is "Just tiredness, can deal with this" and that they "Can live with being tired." HFS provoked mixed responses with some imagining the pain to be very difficult to overcome, "Would have had enough pain - after 3 years. A miserable life" and others feeling that it would not hamper their lives severely, "Difficult but life is still there". Diarrhea was considered to be very challenging to cope with, with participants commenting 
Table I Participant sociodemographics and EQ-5D-3L responses

\begin{tabular}{|c|c|c|c|c|}
\hline & \multicolumn{2}{|l|}{$\begin{array}{l}\text { Participants } \\
(\mathrm{n}=100)\end{array}$} & \multicolumn{2}{|c|}{$\begin{array}{l}\text { UK census (Office of National } \\
\text { Statistics, 20II) }\end{array}$} \\
\hline Sex (male), $\mathrm{n}$ & 45 & & $49 \%$ & \\
\hline Mean age, years (SD) & $41.9(15.9)$ & & 38.2 & \\
\hline \multicolumn{5}{|l|}{ Ethnic background } \\
\hline White & 92 & & $92.1 \%$ & \\
\hline Mixed race & 2 & & $1.9 \%$ & \\
\hline Asian/Asian British & 3 & & $4.0 \%$ & \\
\hline Chinese & 3 & & Not captured & \\
\hline \multicolumn{5}{|l|}{ Main activity } \\
\hline Employed & 65 & & $73 \%$ & \\
\hline Student & 18 & & $5.2 \%$ & \\
\hline Retired & 9 & & $3 \%$ & \\
\hline Stay at home & 6 & & $5.2 \%$ & \\
\hline \multicolumn{5}{|l|}{ Qualifications } \\
\hline Completed university & 47 & & $27 \%$ & \\
\hline $\begin{array}{l}\text { Left school at } 18 \text { with qualifications } \\
\text { or technical/vocation qualification }\end{array}$ & 29 & & $16 \%$ & \\
\hline $\begin{array}{l}\text { Left school at } 16 \text { with qualifications } \\
\text { or no formal qualification }\end{array}$ & 15 & & $51 \%$ & \\
\hline Other & 9 & & $6 \%$ & \\
\hline \multicolumn{5}{|l|}{ Relationship status } \\
\hline Single & 25 & & $36 \%$ & \\
\hline Partnership & 10 & & - & \\
\hline Married & 59 & & $48 \%$ & \\
\hline Divorced/separated & 4 & & $10 \%$ & \\
\hline Widowed & 2 & & $5 \%$ & \\
\hline EQ-5D-3L responses & Moderate (\%) & Extreme (\%) & Moderate (\%) & Extreme (\%) \\
\hline Mobility & 9 & 0 & 18.3 & 0.1 \\
\hline Self-care & 0 & 0 & 4.1 & 0.1 \\
\hline Usual activities & 7 & I & 14.2 & 2.1 \\
\hline Pain/discomfort & 18 & I & 29.2 & 3.8 \\
\hline Anxiety/depression & 9 & I & 19.1 & 1.8 \\
\hline
\end{tabular}

Abbreviation: SD, standard deviation.

"no social life - no real life" and that they "Couldn't deal with this, family not to see".

\section{Modeled health state values}

Results from the multivariable model regression analysis included z-scores, which can be compared against the standard normal distribution. In this analysis, all of the treatment response and $\mathrm{AE}$ health states were shown to be statistically significant in predicting transformed utility. The majority of other covariates were not statistically significant, with the exception of educational qualifications and two EQ-5D-3L variables (usual activity and anxiety/depression).

Table 2 Mean observed utilities for RR-DTC health states and Incremental impact of health states on utilities compared to a base state of stable/no response with no adverse events

\begin{tabular}{|c|c|c|c|c|c|c|}
\hline \multirow[t]{2}{*}{ Parameter } & \multicolumn{2}{|l|}{ Observed mean utility ${ }^{a}$} & \multicolumn{2}{|c|}{ Unadjusted $^{\mathrm{b}}$} & \multicolumn{2}{|c|}{ Adjusted $^{c}$} \\
\hline & $\begin{array}{l}\text { Mean utility } \\
\text { (standard deviation) }\end{array}$ & $\begin{array}{l}95 \% \text { confidence } \\
\text { interval }\end{array}$ & $\begin{array}{l}\text { Utility } \\
\text { value }\end{array}$ & $\begin{array}{l}95 \% \text { confidence } \\
\text { interval }\end{array}$ & $\begin{array}{l}\text { Utility } \\
\text { value }\end{array}$ & $\begin{array}{l}95 \% \text { confidence } \\
\text { interval }\end{array}$ \\
\hline $\begin{array}{l}\text { Base state - stable/no } \\
\text { response }\end{array}$ & $0.80(0.19)$ & $0.77,0.84$ & 0.86 & $0.83,0.90$ & 0.87 & $0.84,0.91$ \\
\hline Response to therapy & $0.86(0.15)$ & $0.83,0.89$ & +0.04 & $0.0 \mathrm{I}, 0.07$ & +0.04 & $0.01,0.07$ \\
\hline Progressive disease & $0.50(0.28)$ & $0.45,0.56$ & -0.37 & $-0.43,-0.31$ & -0.35 & $-0.4 I,-0.29$ \\
\hline Diarrhea & $0.42(0.29)$ & $0.36,0.48$ & -0.48 & $-0.54,-0.43$ & -0.47 & $-0.52,-0.41$ \\
\hline Fatigue & $0.72(0.24)$ & $0.67,0.77$ & -0.08 & $-0.13,-0.04$ & -0.08 & $-0.12,0.04$ \\
\hline Hand and foot syndrome & $0.52(0.30)$ & $0.46,0.58$ & -0.35 & $-0.42,-0.29$ & -0.34 & $-0.40,0.28$ \\
\hline Alopecia & $0.75(0.2 \mathrm{I})$ & $0.71,0.79$ & -0.05 & $-0.09,-0.01$ & -0.05 & $-0.08,0.01$ \\
\hline
\end{tabular}

Notes: aMean observed TTO health state utilities. ${ }^{b}$ Derived from reduced parameter model (health states only). 'Adjusted for educational qualification level and EQ-5D-3L (usual activities and anxiety/depression) ratings using UK norms.

Abbreviations: RR-DTC, radioactive iodine-refractory differentiated thyroid cancer; TTO, time trade off. 
Table 3 Parameter estimates of time trade-off utilities (mixed effects model) including statistically significant health states and sample variables in the overall multivariable model

\begin{tabular}{|c|c|c|c|c|}
\hline Parameter & $\begin{array}{l}\text { Parameter } \\
\text { estimate }\end{array}$ & $\begin{array}{l}\text { Standard } \\
\text { error }\end{array}$ & P-value & $\begin{array}{l}95 \% \text { confidence } \\
\text { intervals }\end{array}$ \\
\hline Intercept & 1.548 & 0.177 & 0.000 & I.2, I.9 \\
\hline \multicolumn{5}{|l|}{ Treatment response health states } \\
\hline \multicolumn{5}{|l|}{ Stable/no response } \\
\hline Response (partial/complete) & 0.406 & 0.139 & 0.004 & $0.13,0.68$ \\
\hline Progressive disease & -1.845 & 0.139 & 0.000 & $-2.12,-1.57$ \\
\hline \multicolumn{5}{|l|}{ Stable treatment response $+\mathrm{AE}$ health states } \\
\hline AE: diarrhea & -2.325 & 0.139 & 0.000 & $-2.6,-2.05$ \\
\hline AE: fatigue & -0.576 & 0.139 & 0.000 & $-0.85,-0.30$ \\
\hline AE: HFS & -1.793 & 0.139 & 0.000 & $-2.07,-1.52$ \\
\hline AE: alopecia & -0.356 & 0.139 & 0.011 & $-0.63,-0.08$ \\
\hline \multicolumn{5}{|l|}{ EQ-5D-3L } \\
\hline EQ-5D: any usual activity problems & -0.871 & 0.403 & 0.031 & $-1.66,-0.08$ \\
\hline EQ-5D: any anxiety/depression problems & 1.238 & 0.465 & 0.008 & $0.33,2.15$ \\
\hline \multicolumn{5}{|l|}{ Qualifications } \\
\hline $\begin{array}{l}\text { Left school at I } 8 \text { with qualifications } \\
\text { or technical/vocational qualifications }\end{array}$ & 0.470 & 0.254 & 0.064 & $-0.33,0.97$ \\
\hline $\begin{array}{l}\text { Left school at } 16 \text { with qualifications } \\
\text { or no formal qualifications }\end{array}$ & 0.861 & 0.335 & 0.010 & $0.20,1.52$ \\
\hline
\end{tabular}

Abbreviations: $A E$, adverse event; HFS, hand and foot syndrome.

Results from the mixed effect model of the health states and these significant variables are shown in Table 3.

The incremental impact of health states on utilities was then compared to a base state of stable/no response with no AEs (Table 2). Two sets of predicted values are listed, the first based on the health state only model (unadjusted) and the second from an adjusted model (data shown in Table 2). To generate predicted values from the adjusted model, EQ-5D-3L usual activities and anxiety/depression domain and educational qualification variables were set to UK normative values. The presence of a response (partial/ complete) was associated with an increase in the utility of 0.04 irrespective of adjustment. The largest utility decrement was associated with grade III diarrhea, which resulted in a -0.48 decrease in utility in the health state only model (unadjusted) and -0.47 based in the adjusted model. Progressive disease was also associated with a decrease in utility of -0.37 in the health state only model and -0.35 in the adjusted model.

\section{Observed versus predicted utility values}

Observed mean TTO values were compared with the predictions from the health state only model, and the adjusted model, with EQ-5D-3L, and qualification variables set to UK normative values (Table 2). The pattern of results across the health states was consistent across the three sets of utility values.

\section{Discussion}

This study aimed to elicit utility values for the HRQL impact of RR-DTC across different treatment responses and AE health states. The results demonstrated clear differentiation in valuation between health states, with response (partial or complete) to treatment valued as having the least impact on HRQL, and stable/no response plus grade III diarrhea being valued as the most burdensome health state. The $\mathrm{AE}$ with the least associated burden was alopecia (grades I/II), followed by fatigue (grade III). These findings were further supported by the alignment of findings across the VAS and TTO results and the consistency of the values produced during the regression analysis with the untransformed data.

An important observation is the comparatively large difference between stable/no response and progressive disease states when compared with previous vignette studies in other malignancies..$^{35,36}$ This is likely to be due to relatively high utility of the stable/no response health state. This interpretation is consistent with views expressed by the clinical experts consulted during the development of the health states and published DTC management guidelines. ${ }^{37}$ The change from stable/no response to disease progression is more substantial as a result, representing a transition from a "positive" state to a "negative" state. In DTC clinical practice, a transition from a progressive disease to a stable disease state represents an important benefit as a subset of patients, even some with quite advanced disease, will live with and die with, not 
from, their DTC. However, although disease stabilization is an important treatment goal in DTC, analyses show that patients with responsive disease are likely to experience a longer progression-free survival than those with stable disease. ${ }^{38}$ These landmark analyses are not without some bias, yet nonetheless, if a lack of progression is considered to be a positive situation, a response is at least likely to prolong this state.

Another observation is the magnitude of impact of certain AEs. Stable/no response plus grade III diarrhea was valued as the most burdensome health state with a mean observed utility of 0.42 . However, the values for the diarrhea health state are lower than previous estimates from vignette studies conducted in different malignancies. For example, a similar vignette study in metastatic renal cell carcinoma reported a mean utility of 0.53 for grade III diarrhea. ${ }^{39}$ Similarly, studies in metastatic breast cancer and non-small-cell lung cancer observed values of 0.61 and 0.61 , respectively. ${ }^{35,36}$ This may reflect differences in the interpretation of AEs and/or variation in the health state descriptions used between studies or differences in the samples and their views regarding the burden of different health states. In addition, two studies used standard gamble rather than TTO methods for health state valuation, ${ }^{35,36}$ which is likely to explain some of the between-study variations in values elicited.

Also of potential interest is the relatively low valued HRQL impact of alopecia (mean observed utility for stable disease with alopecia grades I/II of 0.75 , compared with 0.80 for stable disease without). Given thyroid cancer is more prevalent in women than in men, it is possible that the impact of alopecia as an AE associated with treatment may be valued differently by a RR-DTC patient population than the general public in which the sexes are more equally represented. An exploratory analysis was conducted to examine the impact of sex on preferences around alopecia in this study. The findings were consistent with the hypothesis that women were more concerned with alopecia than men; however, the results were not conclusive. While for many regions societal valuation is required for health utilities for use in economic evaluation of treatments, the potential relevance of alopecia to female DTC patients should not be overlooked, as a patient may consider alopecia to be of higher significance than observed during this study.

\section{Strengths and limitations}

The vignette approach has its limitations, and the data derived from generic measures such as the EQ-5D-3L are preferred by many decisions makers. However, capturing representative
HRQL data from people with rare forms of advanced cancer presents many challenges. The method used in this study to estimate utilities is dependent on the robustness of the methodology used to develop the health state vignettes. To address this, the vignette development was informed by literature review, qualitative work with patients and physicians, and additional rounds of interviews with clinical experts and the general public.

The extreme rarity of RR-DTC also presents an important challenge, as many clinicians lack the treatment experience necessary to accurately describe relevant HRQL factors, and recruiting for a patient-only study lacks feasibility. However, this was overcome by the involvement of clinical experts with 60 years combined experience in treating RR-DTC, who see between two and 30 patients each month. The guidance provided by these experts facilitated the development of accurate and representative disease-related health states, which could undergo valuation by members of the general public. This valuation by the general public is also beneficial, as it considers the priorities of a wider society rather than just those held by a small subgroup of patients. This methodology also meets the requirements for normalization and subsequent health economic decision models informing HTA. The vignette content was also structured around the domains of the EQ-5D$3 \mathrm{~L}$ and SF-6D in order to ensure that the main aspects of HRQL were included rather than just those directly affected by RR-DTC. Therefore, the approach was used to place the problems associated with RR-DTC, its treatments, and the side effects in a more general health context, thus facilitating comparison with other conditions.

The small sample size in the valuation may be a limitation that could affect the representativeness of the results for the general UK population. Some evidence from the EQ-5D-3L data suggest that the sample were relatively healthier than the UK general population. ${ }^{34}$ When compared to the Office of National Statistics census data, ${ }^{33}$ students/university completers and married people were overrepresented in the current study. However, any effect on the mean utilities elicited from this sample was explored in regression analysis, with predicted utility values adjusted for significant sample variables to bring the observed values closer to those expected from a more normative UK sample.

The health states developed and valued in this study limited the valuation of each AE to the context of stable/no response and did not evaluate the impact of AEs when combined with other treatment responses. However, the predictive values offer the potential to estimate the impact of AEs in other health state combinations. It is plausible that the burden 
of AEs might be lower for people who were experiencing a response to treatment, and further qualitative work would be needed to assess the adaptation required. A useful source for the implementation of utilities in cost-effectiveness models is the NICE guidance document, which discusses the adjusting and combining of health state utility values. ${ }^{32}$

During analysis, utility point estimates were devised using three different methods, mean values calculated directly from the observed TTO data, unadjusted values modeled by regression analysis, and adjusted values modeled using UK normative values for certain sample variables. Although the pattern of results was consistent across the analyses, the regression analysis generally yielded results that were slightly higher than those directly calculated from the observed TTO data. As per with mapping to estimate health-related utility, the appropriate model type differs depending on the dataset and how it is applied. Standard econometric and statistical techniques and judgement based on prior knowledge of the clinical relationship between variables should be used to inform model selection and application. The properties of the sample dataset should be used to inform model selection, and a justification should be provided explaining why the selected regression model was chosen. ${ }^{40}$

\section{Conclusion}

In conclusion, the RR-DTC treatment response and associated $\mathrm{AE}$ health states developed and evaluated during this study show clear differentiation. Utility values demonstrated that the treatment response (partial or complete) health state was valued as having the least negative impact on HRQL, and stable/no response plus grade III diarrhea was valued as the most burdensome health state. The order and magnitude of HRQL impact reflected by these utility values were consistent with clinical opinion. The values reported in this study are suitable for use in cost-effectiveness evaluations for new treatments in RR-DTC.

\section{Acknowledgments}

This study was funded by Eisai Inc. The authors would like to thank the following people for their contributions to this research: the clinicians and participants involved in the study, the trained TTO interviewers who assisted with recruitment and data collection, and Sarah Corden for editorial assistance in preparing the manuscript.

\section{Disclosure}

The study was funded by Eisai Inc, who commissioned ICON plc to conduct the work. Corey L Pelletier, Gabriel Tremblay, and Anna Forsythe are employees of Eisai, and Hayley M de Freitas is an employee of ICON plc. Beth A Fordham, Cicely Kerr, and Karissa Johnston were also employees of ICON plc during the time the study was conducted. Andrew $\mathrm{J}$ Lloyd is a consultant for ICON plc. Ezra EW Cohen has undergone paid consulting for Eisai, Bayer, and AstraZeneca. Bryan McIver has no relevant conflicts of interest for the past 2 years, he underwent paid consulting for Eisai in 2012. The authors report no other conflicts of interest in this work.

\section{References}

1. Borget I, Corone C, Nocaudie M, et al. Sick leave for follow-up control in thyroid cancer patients: comparison between stimulation with thyrogen and thyroid hormone withdrawal. Eur J Endocrinol. 2007; 156:531-538.

2. Kilfoy BA, Zheng T, Holford TR, et al. International patterns and trends in thyroid cancer incidence, 1973-2002. Cancer Causes Control. 2009;20(5):525-531

3. Surveillance, Epidemiology, and End Results Program. SEER Stat Fact Sheets: Thyroid Cancer. Available from: http://seer.cancer.gov/ statfacts/html/thyro.html. Accessed May 29, 2015.

4. Duntas L, Biondi B. Short-term hypothyroidism after Levothyroxinewithdrawal in patients with differentiated thyroid cancer: clinical and quality of life consequences. Eur J Endocrinol. 2007;156:13-19.

5. Almeida J, Vartanian J, Kowalski L. Clinical predictors of quality of life in patients with initial differentiated thyroid cancers. Arch Otolaryngol Head Neck Surg. 2009;135(4):342-346.

6. Durante C, Haddy N, Baudin E, et al. Long-term outcome of 444 patients with distant metastases from papillary and follicular thyroid carcinoma: benefits and limits of radioiodine therapy. J Clin Endocrinol Metab. 2006;91:2892-2899.

7. Rahib L, Smith BD, Aizenberg R, Rosenzweig AB, Fleshman JM, Matrisian LM. Projecting cancer incidence and deaths to 2030: the unexpected burden of thyroid, liver, and pancreas cancers in the United States. Cancer Res. 2014;74(11):2913-2921.

8. Schultz P, Stava C, Vassilopoulou-Sellin R. Health profiles and quality of life of 518 survivors of thyroid cancer. Head Neck. 2003;25(5):349-356.

9. Hoftijzer H, Heemstra K, Corssmit E, van der Klaauw A, Romijn J, Smit J. Quality of life in cured patients with differentiated thyroid carcinoma. J Clin Endocrinol Metab. 2008;93(1):200-203.

10. Botella-Carretero J, Galan J, Caballero C, Sancho J, Escobar-Morreale HF. Quality of life and psychometric functionality in patients with differentiated thyroid carcinoma. Endocr Relat Cancer. 2003;10:601-610.

11. Lee JI, Kim SH, Tan AH, et al. Decreased health-related quality of life in disease-free survivors of differentiated thyroid cancer in Korea. Health Qual Life Outcomes. 2010;8:101.

12. Tan L, Nan L, Thumboo J, Sundram F, Tan L, Rhinological and Otological Society, Inc. Health-related quality of life in thyroid cancer survivors. Laryngoscope. 2007;117:507-510.

13. Pacini F, Ito Y, Luster M, Pitoia F, Robinson B, Wirth L. Radioactive iodine-refractory differentiated thyroid cancer: unmet needs and future directions. Expert Rev Endocrinol Metab. 2012;7(5):541-554.

14. Sherman SI. Cytotoxic chemotherapy for differentiated thyroid carcinoma. Clin Oncol (R Coll Radiol). 2010;22(6):464-468.

15. EMA. EMA Public Summary of Opinion on Orphan Designation. Sorafenib Tosylate for the Treatment of Follicular Thyroid Cancer; 2014. Available from: http://www.ema.europa.eu/docs/en_GB/ document_library/Orphan_designation/2013/11/WC500156178.pdf. Accessed December 4, 2014.

16. FDA News Release. FDA Approves Nexavar to Treat Type of Thyroid Cancer; 2013. Available from: http://www.fda.gov/NewsEvents/ Newsroom/PressAnnouncements/ucm376443.htm. Accessed November 18, 2014. 
17. FDA News Release. FDA Approves Lenvima for a Type of Thyroid Cancer; 2015. Available from: http://www.fda.gov/NewsEvents/ Newsroom/PressAnnouncements/ucm434288.htm. Accessed March 17, 2015.

18. Wirth LJ. Considerations for initiating treatment in advanced thyroid cancer. Clin Adv Heamtol Oncol. 2014;12(suppl 14):19-21.

19. Gallop K, Kerr C, Simmons S, McIver B, Cohen EEW. A qualitative evaluation of the validity of published health utilities and generic health utility measures for capturing health-related quality of life (HRQL) impact of differentiated thyroid cancer (DTC) at different treatment phases. Qual Life Res. 2015;24(2):325-338.

20. Mernagh P, Campbell S, Dietlein M, Luster M, Mazzaferri E, Weston A. Cost effectiveness of using recombinant human TSH prior to radioiodine ablation for thyroid cancer, compared with treating patients in a hypothyroid state: the German perspective. Eur J Endocrinol. 2006; 155:405-414.

21. Mernagh P, Suebwongpat A, Silverberg J, Weston A. Cost effectiveness of using recombinant human thyroid-stimulating hormone before radioiodine ablation for thyroid cancer: the Canadian perspective. Value Health. 2010;13(2):180-187.

22. Wang T, Cheung K, Mehta P, Roman S, Walker H, Sosa J. Recombinant human thyrotropin versus thyroxine withdrawal for radioiodine ablation in patients with low-risk differentiated thyroid cancer in the United States. J Clin Endocrinol Metab. 2010;95(4):1672-1680.

23. Kebebew E, Duh Q, Clark O. Total thyroidectomy or thyroid lobectomy in patients with low-risk differentiated thyroid cancer: surgical decision analysis of a controversy using a mathematical model. World J Surgery. 2000;24:1295-1302.

24. NICE. Process and Methods Guides: Guide to the Methods of Technology Appraisal; 2013. Available from: http://www.nice.org.uk/article/pmg9/ resources/non-guidance-guide-to-the-methods-of-technology-appraisal2013-pdf. Accessed May 29, 2015.

25. Stajduhar KI, Neithercut J, Chu E, et al. Thyroid cancer: patients' experiences of receiving iodine-131 therapy. Oncol Nurs Forum. 2000;27(8): 1213-1218.

26. Sawka AM, Goldstein DP, Brierley JD, et al. The impact of thyroid cancer and post-surgical radioactive iodine treatment on the lives of thyroid cancer survivors: a qualitative study. PLoS One. 2009;4(1):e4191.

27. EuroQol Group. EuroQol - a new facility for the measurement of health-related quality of life. Health Policy. 1990;16:199-208.

28. Brazier J, Usherwood T, Harper R, Thomas K. Deriving a preferencebased single index from the UK SF-36 Health Survey. J Clin Epidemiol. 1998;51:1115-1128.
29. Eisenhauer E, Therasseb P, Bogaertsc J, et al. New response evaluation criteria in solid tumours: revised RECIST guideline (version 1.1). Eur J Cancer. 2009;45:228-247.

30. Cancer Therapy Evaluation Program. Common Toxicity Criteria v.2.0; 2009. Available from: http://www.eortc.be/services/doc/ctc/ctcv20_430-992.pdf. Accessed November 18, 2014.

31. Torrance GW, Thomas WH, Sackett DL. A utility maximization model for evaluation of health care programs. Health Serv Res. 1972; $7(2): 118-133$.

32. Ara R, Wailoo AJ. NICE Decision Support Unit (DSU) Technical Support Document 12: The Use of Health State Utility Values in Decision Models; 2011. Available from: http://www.nicedsu.org.uk/TSD12\%20 Utilities\%20in\%20modelling\%20FINAL.pdf. Accessed December 4, 2014.

33. Office for National Statistics (ONS). Census for England and Wales; 2011. Available from: http://www.ons.gov.uk/census/index.html. Accessed December 4, 2014.

34. Kind P, Dolan P, Gudex C, Williams A. Variations in population health status: results from a United Kingdom national questionnaire survey. BMJ. 1993;316:736-741.

35. Lloyd A, Nafees B, Narewska J, Dewilde S, Watkins J. Health state utilities for metastatic breast cancer. Br J Cancer. 2006;95:683-690.

36. Nafees B, Stafford M, Gavriel S, Bhalla S, Watkins J. Health state utilities for non-small cell lung cancer. Health Qual Life Outcomes. 2008;6:84.

37. Cooper DS, Doherty GM, Haugen BR, et al. Revised American Thyroid Association management guidelines for patients with thyroid nodules and differentiated thyroid cancer. Thyroid. 2009;19:1167-1214.

38. Brose MS, Nutting CM, Jarzab B, et al; DECISION Investigators. Sorafenib in radioactive iodine-refractory, locally advanced or metastatic differentiated thyroid cancer: a randomised, double-blind, phase 3 trial. Lancet. 2014;384:319-328.

39. Swinburn P, Lloyd A, Nathan P, Choueiri TK, Cella D, Neary MP. Elicitation of health state utilities in metastatic renal cell carcinoma Curr Med Res Opin. 2010;26(5):1091-1096.

40. Longworth L, Rowen D. NICE Decision Support Unit (DSU) Support Document 10: The Use of Mapping Methods to Estimate Health State Utility Values; 2011. Available from: http://www.nicedsu.org. uk/TSD\%2010\%20mapping\%20FINAL.pdf. Accessed December 8, 2014. 


\section{Supplementary materials Final health states with descriptions Stable/no response}

The differences in health state descriptions were highlighted by underlining key text in each health state that differentiated one state from another.

- You are currently undergoing a treatment for a long-term

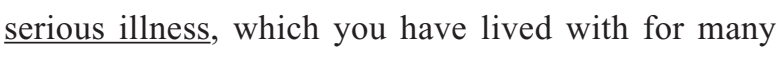
years.

- Your illness is not getting better with treatment, but it is not currently getting any worse.

- You sometimes have discomfort in your neck related to trouble swallowing or mild swelling in your neck.

- You have a dry mouth, which causes you discomfort especially on waking, when talking for a long time or eating. You need to regularly drink water to relieve this sensation and have to carry water with you to do so.

- You sometimes feel tired and lack energy.

- You are able to walk around as normal and you are able to wash and dress yourself.

- You are able to perform usual activities such as working but you may need to rest, which can limit your social activities.

- You sometimes experience pain.

- You are relieved that your illness is currently not getting any worse, but you are uncertain how long this will last and you are worried about the future.

- Your mood can often feel low, which causes strain on your closest relationships.

\section{Response}

- You are currently undergoing a treatment for a long-term serious illness, which you have lived with for many years.

- Your illness is currently showing some signs that it is responding to treatment, so getting better.

- You sometimes have discomfort in your neck related to trouble swallowing or mild swelling in your neck.

- You have a dry mouth, which causes you discomfort especially on waking, when talking for a long time or eating. You need to regularly drink water to relieve this sensation and have to carry water with you to do so.

- You sometimes feel tired and lack energy.

- You are able to walk around as normal and you are able to wash and dress yourself.

- You are able to perform usual activities such as working but you may need to rest, which can limit your social activities.

- You sometimes experience pain.
- You are relieved and happy your illness is getting better but are uncertain how long this will last, which causes you some worry about the future.

- Your mood can sometimes feel low, which can cause $\underline{\text { strain on your closest relationships. }}$

\section{Progressive disease}

- You are currently undergoing a treatment for a long-term serious illness, which you have lived with for many years.

- Your illness is getting worse despite treatment.

- You sometimes have discomfort in your neck related to trouble swallowing or mild swelling in your neck.

- You have a dry mouth, which causes you discomfort especially on waking, when talking for a long time or eating. You need to regularly drink water to relieve this sensation and have to carry water with you to do so.

- You are often tired and lack energy.

- You have some problems walking and you sometimes find it hard to wash and dress yourself.

- You are not able to perform some of your usual activities such as working full time and you often need to rest, which limits your social activities.

- You often experience pain.

- You are very distressed that current treatment is not working and your illness is getting worse. You feel fearful about the future.

- You often have low mood and may have feelings of depression. This causes significant strain on your closest relationships.

\section{Stable + grade 3 diarrhea}

- You are currently undergoing a treatment for a long-term serious illness, which you have lived with for many years.

- Your illness is not getting better with treatment, but it is not currently getting any worse.

- You sometimes have discomfort in your neck related to trouble swallowing or mild swelling in your neck.

- You have a dry mouth, which causes you discomfort especially on waking, when talking for a long time or eating. You need to regularly drink water to relieve this sensation and have to carry water with you to do so.

- You experience $\geq 7$ episodes of diarrhea a day. You experience diarrhea during the night, which can interrupt your sleep. You can occasionally lose control over your bowel movements.

- You feel tired and lack energy. 
- You are able to walk around as normal, but because of your diarrhea you do not want to be far from a toilet. You are able to wash and dress yourself.

- You are not able to perform some of your usual activities such as working full time because you need to rest and you have to plan your day to be close to a toilet. You may prefer to stay at home or close to home. This has a large impact upon your social activities.

- You experience mild pain, including stomach cramps.

- You are relieved that your illness is currently not getting any worse but you are uncertain how long this will last and you are worried about the future.

- Your mood can often feel low, which causes strain on your closest relationships.

\section{Stable + grade 3 fatigue}

- You are currently undergoing a treatment for a long-term serious illness, which you have lived with for many years.

- Your illness is not getting better with treatment but it is not currently getting any worse.

- You sometimes have discomfort in your neck related to trouble swallowing or mild swelling in your neck.

- You have a dry mouth, which causes you discomfort especially on waking, when talking for a long time or eating. You need to regularly drink water to relieve this sensation and have to carry water with you to do so.

- You feel tired and lack energy most of the time.

- You are able to walk but only for short distances. You are able to wash and dress yourself.

- You are not able to perform some of your usual activities such as working full time. You need to plan your day in order to take regular breaks to rest or sleep more. This significantly limits your time and energy for social activities.

- You sometimes experience pain.

- You are relieved that your illness is currently not getting any worse but you are uncertain how long this will last and you are worried about the future.

- Your mood can often feel low, which causes strain on your closest relationships.

\section{Stable + grade 3 HFS}

- You are currently undergoing a treatment for a long-term serious illness, which you have lived with for many years.

- Your illness is not getting better with treatment, but it is not currently getting any worse.
- You sometimes have discomfort in your neck related to trouble swallowing or mild swelling in your neck.

- You have a dry mouth, which causes you discomfort especially on waking, when talking for a long time or eating. You need to regularly drink water to relieve this sensation and have to carry water with you to do so.

- You experience a skin condition that causes redness, peeling, dryness, blistering, and tenderness on your hands and feet.

- You sometimes feel tired and lack energy.

- You have significant problems with walking and you have some problems with washing and dressing yourself.

- You are able to perform some of your usual activities but have difficulty performing any that involve using your hands or feet. For example, you can experience difficulty using a keyboard, holding a pen, standing, or climbing stairs. This can limit your work and social activities.

- You experience pain, especially in your hands and feet.

- You are relieved that your illness is currently not getting any worse, but you are uncertain how long this will last, and you are worried about the future.

- Your mood can often feel low, which causes strain on your closest relationships.

\section{Stable + grades I or 2 alopecia}

- You are currently undergoing a treatment for a long-term serious illness, which you have lived with for many years.

- Your illness is not getting better with treatment, but it is not currently getting any worse.

- You sometimes have discomfort in your neck related to trouble swallowing or mild swelling in your neck.

- You have a dry mouth, which causes you discomfort especially on waking, when talking for a long time or eating. You need to regularly drink water to relieve this sensation and have to carry water with you to do so.

- You have lost some or all of your hair.

- You sometimes feel tired and lack energy.

- You are able to walk around as normal, and you are able to wash and dress yourself.

- You are able to perform usual activities such as working, but you may need to rest, which can limit your social activities.

- You sometimes experience pain.

- You are relieved that your illness is currently not getting any worse, but you are uncertain how long this will last and you are worried about the future.

- Your mood can often feel low, which causes strain on your closest relationships. 


\section{Publish your work in this journal}

Patient Preference and Adherence is an international, peer-reviewed, open access journal that focuses on the growing importance of patient preference and adherence throughout the therapeutic continuum. Patient satisfaction, acceptability, quality of life, compliance, persistence and their role in developing new therapeutic modalities and compounds to optimize

clinical outcomes for existing disease states are major areas of interest for the journal. This journal has been accepted for indexing on PubMed Central. The manuscript management system is completely online and includes a very quick and fair peer-review system, which is all easy to use. Visit http://www. dovepress.com/testimonials.php to read real quotes from published authors.

Submit your manuscript here: http://www.dovepress.com/patient-preference-and-adherence-journal 